\title{
Performance Evaluation of OFDM System with Insufficient CP Using LMS Equalizer under Harsh Multipath Conditions
}

\author{
Abolqassem Fakher \\ Department of Electrical \\ Engineering \\ University of Basrah \\ Iraq - Basrah \\ abolqassem44@yahoo.com
}

\author{
Falih M. Alnahwi \\ Department of Electrical \\ Engineering \\ University of Basrah \\ Iraq - Basrah \\ fmmm1983@gmal.com
}

\author{
Majid A. Alwan \\ Department of Electrical \\ Engineering \\ University of Basrah \\ Iraq - Basrah \\ altimimee@yahoo.com
}

\begin{abstract}
This paper presents an insufficient cyclic prefix (CP) Orthogonal Frequency Division Multiplexing (OFDM) system with equalizer whose coefficients are calculated using Least Mean Square (LMS) algorithm. The OFDM signal is passed through a channel with four multipath signals which cause the OFDM signal to be under high inter-symbol interference (ISI) and inter-carrier interference (ICI).8-QAM and 16-QAM digital modulation techniques are used to evaluate the performance of the proposed system. The simulation results have accentuated the high performance of the LMS equalizer via comparing its Bit Error Rate (BER) and constellation diagram with those of the Minimum Mean Square Error and Zero Forcing equalizers. Moreover, the results also reveal that the LMS equalizer provides BER performance close to that of the OFDM system with a hypothetical sufficient CP.
\end{abstract}

Index Terms - multi-carrier modulation, frequency selective fading, Least Mean Square, Orthogonal Frequency Division Multiplexing, subcarriers.

\section{Introduction:}

Orthogonal Frequency Division Multiplexing (OFDM) is the most convenient method that provides sensible immunity against the multipath fading impairment [1]. OFDM could be considered as a special case of multi-carrier modulation (MCM) where the frequency division multiplexing FDM is applied to a large number of subcarriers. OFDM is spectrally efficient and robust transmission technique for broadband digital communication systems, and it is effective against many channel adversities such as the frequency selective fading and impulse noise. OFDM has the ability to convert the wideband frequency selective fading channel into a series of flat fading with narrow band channel. In OFDM technology, Inverse Fast Fourier Transform IFFT is utilized to divide the available spectrum into many subcarriers, but this sub channels undergo Inter Symbol Interference (ISI) and Inter Carrier Interference (ICI) that impact the system performance significantly. Therefore, a cyclic prefix $(\mathrm{CP})$, which is also called the guard time, is inserted between every two adjacent OFDM symbols to avoid the aforementioned types of 
interference [2]. In the receiver side, Fast Fourier Transform (FFT) is used to recover the original signal [3-8].

Increasing the length of the $\mathrm{CP}$ results in perfect elimination for the ISI; however, this increment requires reduction in the data rate (for the same signal bandwidth) and noticeable reduction in the spectral efficiency [9]. On the other hand, short CP (insufficient CP) drops the system immunity against the ISI and ICI but with the advantage of satisfactory spectral efficiency. The compromise between these two solutions is acquired by using insufficient $\mathrm{CP}$ and inserting equalizer at the receiver to compensate the residual effect of the channel impairment that the $\mathrm{CP}$ could not eliminate. Many attempts were proposed to design equalizers to minimize the multipath influence on the received OFDM signal. In [10], a frequency equalizer (FEQ) was proposed by utilizing the presence of null side sub-carriers and a redundancy of CP. In [11], a Radial Basic Function (RBF) neural network was used as a channel equalization technique. A tentative decision was suggested as an iterative method for equalization purposes [12]. In addition, minimum mean square error (MMSE) algorithm was devoted for equalization process [13]. The solution of MMSE was optimized in [14] to obtain more robust OFDM equalization. In [15], a multipath division SC/MMSE was proposed as a turbo equalizer. Finally, a bi-directional Malgorithm (BDMA) for equalization purposes is presented in [16]. All the previous works have been done to improve the system performance when insufficient $\mathrm{CP}$ is used.

This paper elucidating the superiority of the Least Mean Square (LMS) algorithm in calculating suitable OFDM equalizer coefficients to properly combat the multipath fading presented in a certain outdoor channel. Four multipath signals are considered to be presented in the channel, and the simulation is re-executed at different values of signal-to-noise ratio (SNR). The channel is mathematically modeled under the consideration of the ISI and ICI effects. As a matter of comparison, the performance of the proposed system is compared with that of the MMSE and the Zero Forcing (ZF) equalizers by demonstrating the system Bit Error Rate (BER) and the constellation diagram of 8-QAM and 16QAM digital modulation techniques.

\section{System Modeling:}

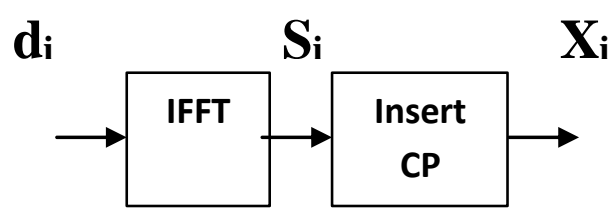

Fig.1 System model in the transmitter side

Fig. 1 illustrates the model of the OFDM transmitter. Let's consider that $\mathrm{d}_{\mathrm{i}}=[\mathrm{di}(0)----\mathrm{di}(\mathrm{N}-$ 1) $]^{\mathrm{T}}$ is an IFFT input that represents an OFDM symbol in the frequency domain. $\mathrm{S}_{\mathrm{i}}=[\mathrm{Si}(0)$---$\mathrm{Si}(\mathrm{N}-1)]$ is the N-point IFFT [17], and it could be represented by $\mathrm{S}_{\mathrm{i}}=\mathrm{W}^{\mathrm{H}} \mathrm{d}_{\mathrm{i}}$, where $\mathrm{W}$ represents the IFFT coefficients and the superscript $\mathrm{H}$ denotes the Hermitian matrix. $x_{i}$ represents the OFDM signal 
after adding $\mathrm{CP}$ with length $\mathrm{m}$ to $\mathrm{S}_{\mathrm{i}}$. Thus, $\mathrm{x}_{\mathrm{i}}=\left[\mathrm{S}_{\mathrm{i}}(\mathrm{N}-\mathrm{m})\right.$ - - - $\left.\mathrm{S}_{\mathrm{i}}(\mathrm{N}-1) \quad \mathrm{S}_{\mathrm{i}}(0)-\cdots \mathrm{S}_{\mathrm{i}}(\mathrm{N}-1)\right]$.

The channel model including the channel noise and the multipath effect is demonstrated in Fig. 2.

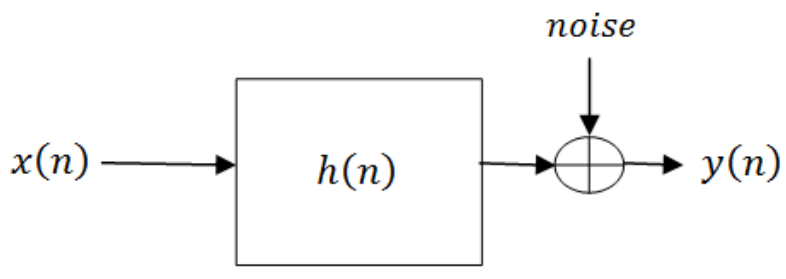

Fig.2 Input data through the channel

The transmitted symbol $\mathrm{x}_{\mathrm{i}}(\mathrm{n})$ is passed through a Single Input Single Output (SISO) system that has $\mathrm{N}$ symbols [17]. The channel impulse response $h(n)$ with length $\mathrm{L}_{\mathrm{h}}$ has been modeled using a standard "delay profile tables" [18]. Thus, the received signal $y_{i}(n)$ is given by:

$y_{i}(n)=h(n) * x(n)=\sum_{v=0}^{L_{h-1}} h(v) x(n-v)$

Each present symbol of $x(n)$ will appear as ISI on the other symbol so that the effect of the previous symbol (i-1) on the present $\mathrm{i}^{\text {th }}$ symbol is given by:

$$
\begin{aligned}
y I S I(n)= & \sum_{v=L+1+n}^{L_{h-1}} h(v) x(n-v) \\
& \text { with } 0 \leq n \leq L_{h}-L-2
\end{aligned}
$$

By considering the ISI begins when the first OFDM symbol is finished until the OFDM channel is finished. Similarly, the ICI affect can be written as:

$$
y \operatorname{ICI}(n)=-\sum_{\substack{v=L+1+n \\-v) \bmod N)}}^{L_{h-1}} h(v) x((n
$$

Therefore, the received signal at the ith symbol at the channel output can be given by [19]:

$$
\mathbf{y}_{\mathbf{i}}=\mathbf{S i}_{\mathbf{i}} \mathbf{H}-\left(\mathbf{S}_{\mathbf{i}} A-\mathbf{S}_{\mathbf{i}} \mathbf{B}\right)+\mathbf{u}_{\mathbf{i}}
$$

where $u_{i}$ represents the received noise, $H$ represents the signal part that is free of noise, A denotes the ICI effect, and B denotes the ISI effect. It is worth to mention that if the channel order $L_{h}$ is less than CP length the ISI and ICI part will equal to zero. This means that the received signal could be demodulated directly using FFT algorithm without the need for any form of equalization [20-21]. The representation of matrix $\mathrm{H}, \mathrm{A}$, and $\mathrm{B}$ is given by:

$H$

$=\left[\begin{array}{cccccccc}h_{0} & 0 & \ldots & 0 & h_{L-1} & h_{L-2} & \ldots & h_{1} \\ h_{1} & h_{0} & 0 & \ldots & 0 & h_{L-1} & \ldots & h_{2} \\ \vdots & \ddots & \ddots & \ddots & \ddots & \ddots & \ddots & \vdots \\ h_{L-1} & h_{L-2} & \ldots & h_{0} & 0 & \ldots & \ldots & 0 \\ 0 & h_{L-1} & h_{L-2} & \ldots & h_{0} & 0 & \ldots & 0 \\ \vdots & \ddots & \ddots & \ddots & \ddots & \ddots & \ddots & \vdots \\ 0 & \ldots & 0 & h_{L-1} & h_{L-2} & \ldots & \ldots & h_{0}\end{array}\right]$

$A$

$=\left[\begin{array}{ccccccccc}0 & \ldots & h_{L-1} & \ldots & \ldots & h_{v+1} & 0 & \ldots & 0 \\ 0 & \ldots & 0 & h_{L-1} & \ldots & h_{v+2} & 0 & \ldots & 0 \\ \vdots & \ddots & \ddots & \ddots & \ddots & \ddots & \ddots & \ddots & \vdots \\ 0 & \ldots & 0 & \ldots & 0 & h_{L-1} & 0 & \ldots & 0 \\ 0 & \ldots & 0 & \ldots & \ldots & 0 & 0 & \ldots & 0 \\ \vdots & \ddots & \ddots & \ddots & \ddots & \ddots & \ddots & \ddots & \vdots \\ 0 & \ldots & 0 & \ldots & \ldots & 0 & 0 & \ldots & 0\end{array}\right]$

$$
B=\left[\begin{array}{ccccccc}
0 & \ldots & 0 & h_{L-1} & \ldots & \ldots & h_{v+1} \\
0 & \ldots & 0 & 0 & h_{L-1} & \ldots & h_{v+2} \\
\vdots & \ddots & \ddots & \ddots & \ddots & \ddots & \vdots \\
0 & \ldots & 0 & \ldots & \ldots & 0 & h_{L-1} \\
0 & \ldots & 0 & \ldots & \ldots & 0 & 0 \\
\vdots & \ddots & \ddots & \ddots & \ddots & \ddots & \ddots \\
0 & \ldots & 0 & \ldots & \ldots & 0 & 0
\end{array}\right]
$$

with $0 \leq n \leq L_{h}-L-2$ 


\section{The Proposed LMS Equalizer:}

The structure of the Least Mean Square (LMS) algorithm is shown in Fig. 3. LMS algorithm is a type of adaptive filter that is used to find the equalizer filter coefficients. The advantages of this algorithm are concluded by its robustness against the channel variations and its

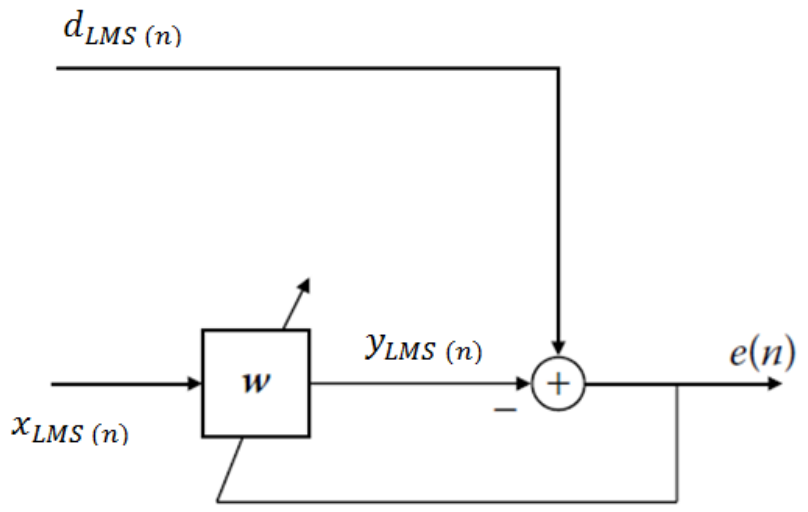

Fig.3 LMS algorithm

low computational complexity. $x_{L M S}(n)$ is a training signal after passing it through the channel, while $d_{L M S}(n)$ is the original training data. $y_{L M S}(n)$ is the output of the adaptive equalizer, and $e(n)$ is the error between the output signal and the desired signal. The weights vector $(\boldsymbol{w})$ of the LMS vector will set to be the equalizer coefficients. LMS is an iterative algorithm whose weights vector is obtained by applying the following equations:

$y_{L M S}(n)=\sum_{k=0}^{M-1} w^{*}(n) x_{L M S}(n-k)$

$e(n)=d_{L M S}(n)-y_{L M S}(n)$

Let $\mathrm{w}(\mathrm{n})$ is the weights vector at step $\mathrm{n}$ [20], so the value of next step $w(n+1)$ is given by: $w(n+1)=w(n)+2 \mu e^{*}(n) x(n)$

Where $(*)$ represented the complex conjugate operator, and $\mu$ represents the step size of the algorithm. The algorithm is stopped once the error signal approaches to zero where the weights vector of the next step is equal to that of the present step.

\section{Simulation Results:}

In this section, the performance of the OFDM system after using the LMS equalizer with a short $\mathrm{CP}$ (insufficient $\mathrm{CP}$ ), then comparing it with that of the Minimum Mean Square Error (MMSE) and Zero Forcing (ZF) equalizers. In addition, an OFDM system with very long CP (sufficient $\mathrm{CP}$ ) is used as a reference for the comparison process.

The simulation process considers the following conditions: sampling time $t_{s}=1 \mu \mathrm{s}$, a fading channel with Gaussian distribution, multipath frequency selective channel has four taps, power delay profile [0 $\left.4 \begin{array}{lll} & 8 & 12\end{array}\right] \mu \mathrm{s}$ (COST 207 model) given in [22]. For the OFDM system, the data length per subcarrier is $\mathrm{N}=128$, and two QAM modulations modulation schemes (8- QAM and 16-QAM) are used to demonstrate the system performance. The insufficient $\mathrm{CP}$ length is selected to be equal to 6 , where as the sufficient $\mathrm{CP}$ length is $40 \%$ of $\mathrm{N}$ which is equal to 52 . Since the block size is 128 and the sampling time is $1 \mu \mathrm{s}$, the overall time of each block is $128 \mu$ s. On the other hand, the time difference between the first and the fourth path is $12 \mu \mathrm{s}$, so the number of samples e=corresponding to the time difference is 12 samples, as a result the 
ISI certainly occurs due to the presence of the insufficient cyclic prefix (6 samples).

\section{A- BER Performance:}

The bit error rate (BER) versus signal-tonoise ratio (SNR) for 8-QAM and 16-QAM are shown in Fig. 4(a) and (b), respectively.

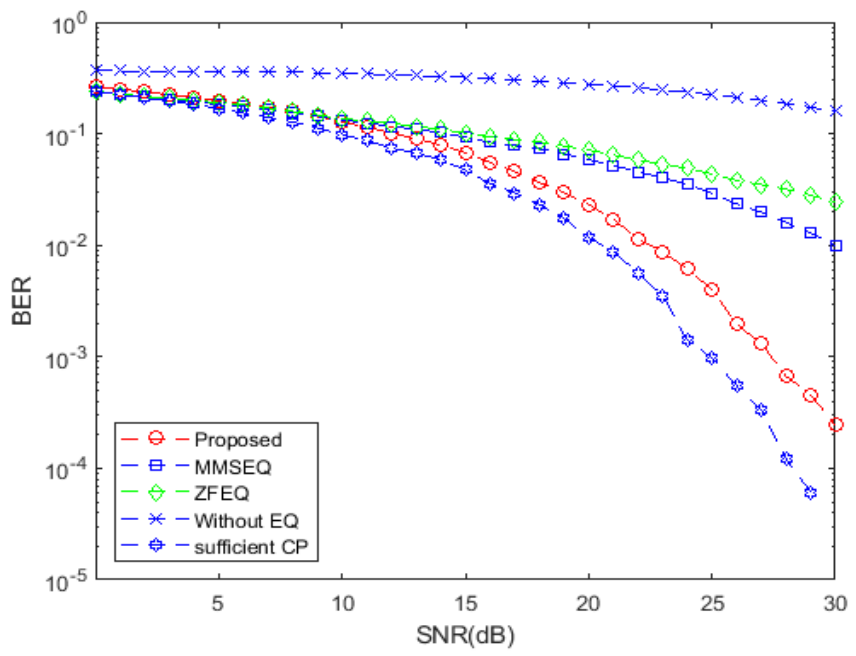

a- Using 8-QAM modulation

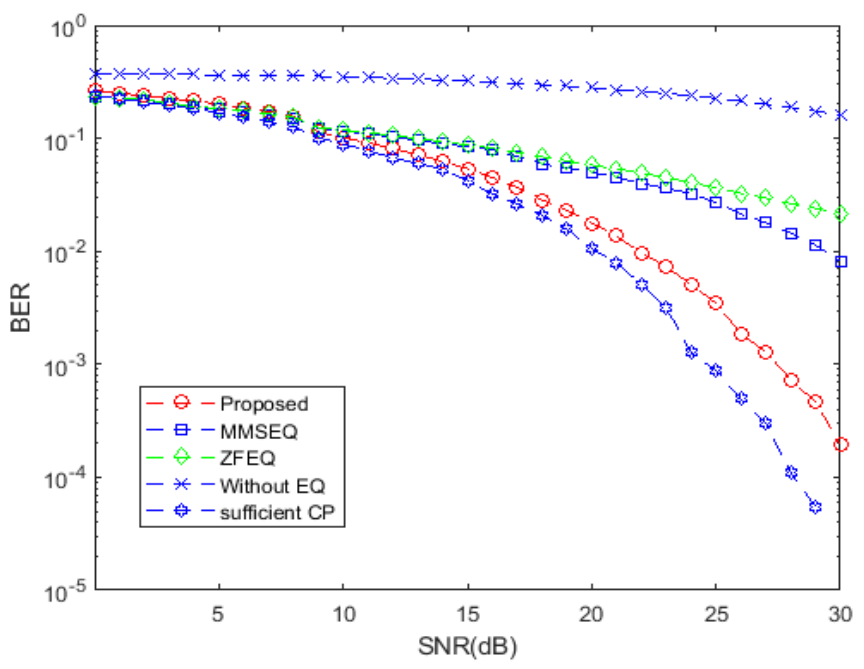

b- Using 16-QAM modulation

Fig.4 BER vs SNR with different types of equalizers. $[13,20]$

The performance of the proposed LMS algorithm is compared with MMSE equalizer, $\mathrm{ZF}$ equalizer, without equalizer, and the hypothetical sufficient
CP transmission. As can be seen in these figures, the performance of insufficient CP LMS algorithm overtakes the performance of the MMSE and ZF and very close to the hypothetical sufficient $\mathrm{CP}$ case.

\section{B- Constellation Diagram:}

To give more insight for the superiority of the LMS over the other techniques, the constellation diagrams of 8-QAM and 16-QAM systems at $\mathrm{SNR}=15 \mathrm{~dB}$ are presented in this subsection. The transmitted data constellation diagram of the 8-QAM and the 16-QAM are given in Fig. 5(a) and (b), respectively. The received signal constellation diagrams without equalizer, ZF equalizer, MMSE equalizer, and the LMS equalizer for 8-QAM and 16-QAM are given in Fig. 6 and Fig. 7, respectively. It is clear that the received data of the LMS equalizer are well concentrated in such a way that the receiver can easily distinguish the different states of the received data.

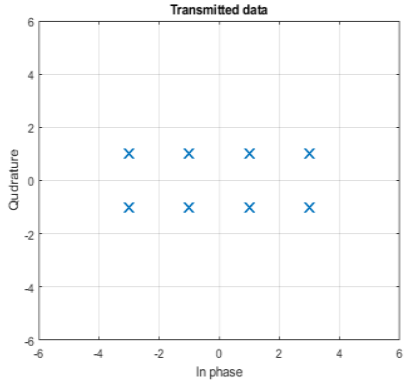

a

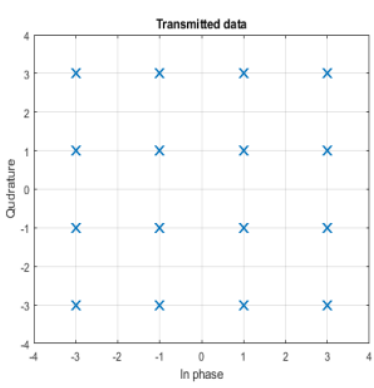

b
Fig.5 the constellation diagrams of transmitted data of a-8-QAM and b-16-QAM systems 


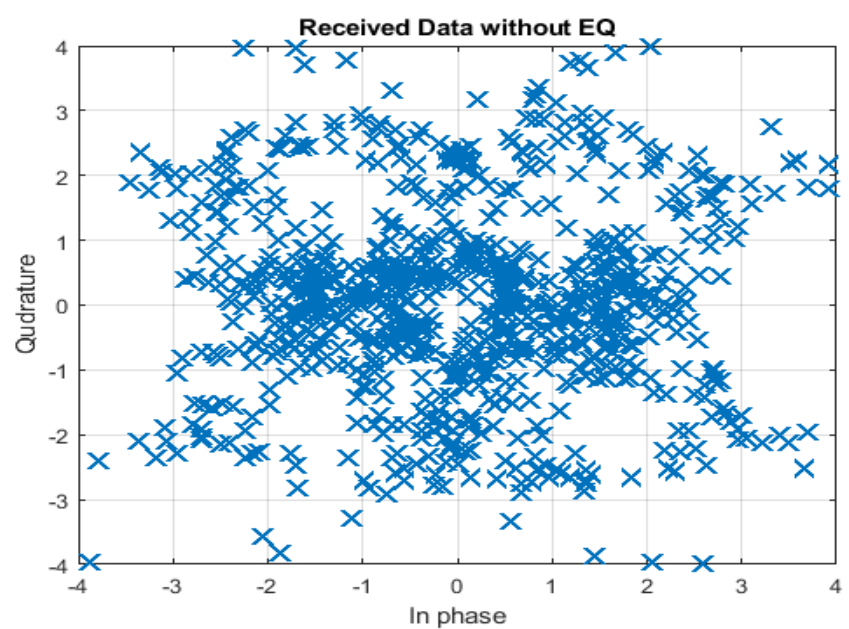

a- received data without using equalizer

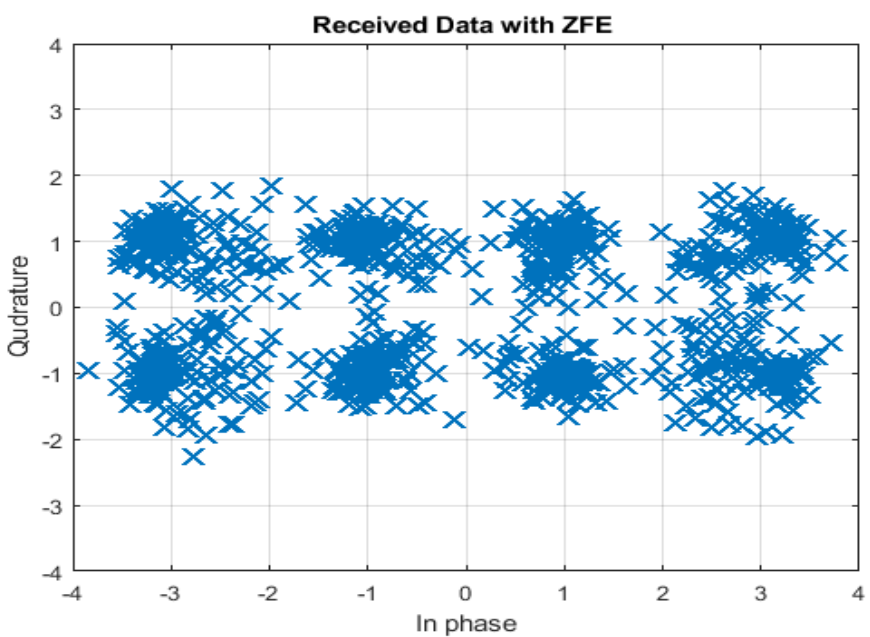

b- received data with $\mathrm{ZF}$ equalizer

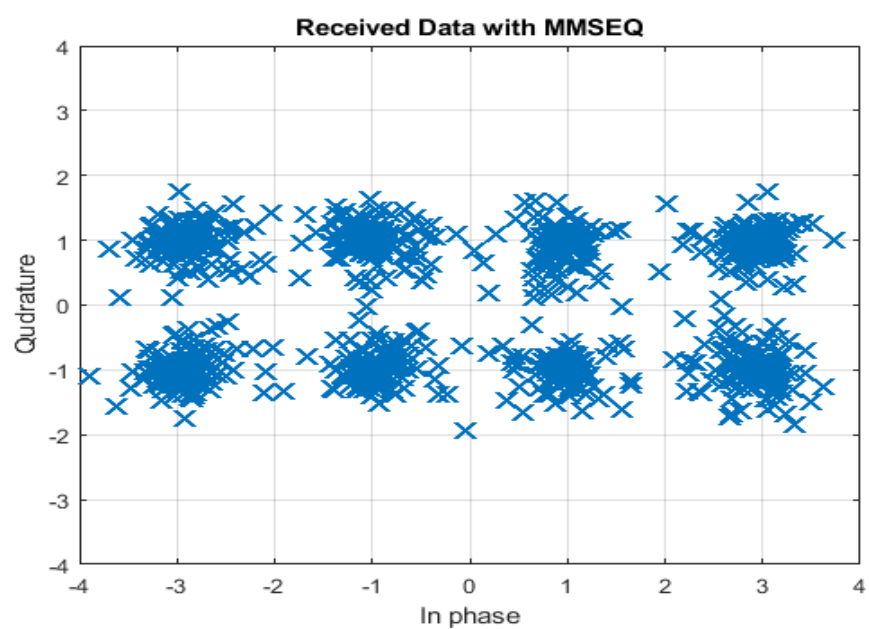

c- received data with MMSE equilzer

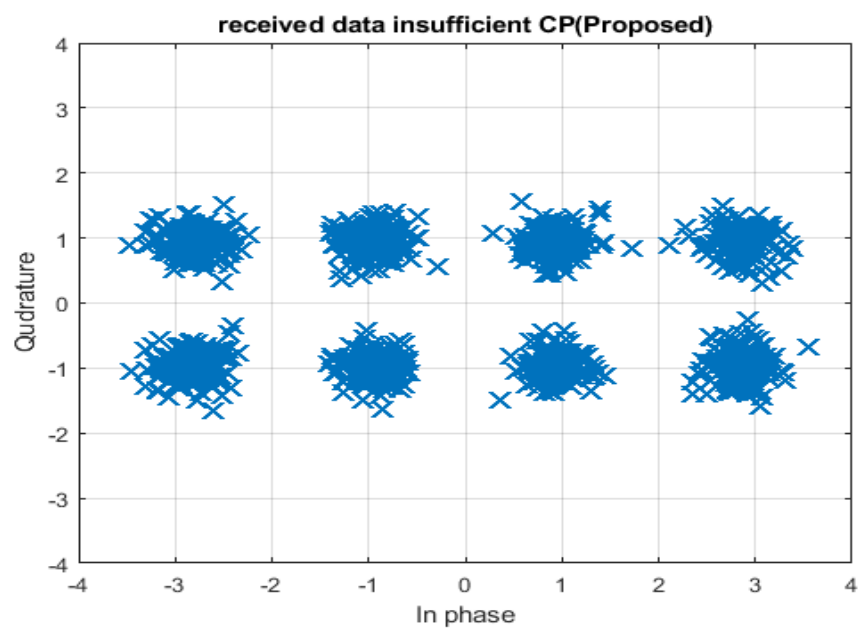

d- recived data with LMS equlizer

Fig. 6 Constellation diagram of 8-QAM received data under different types of equalization techniques.

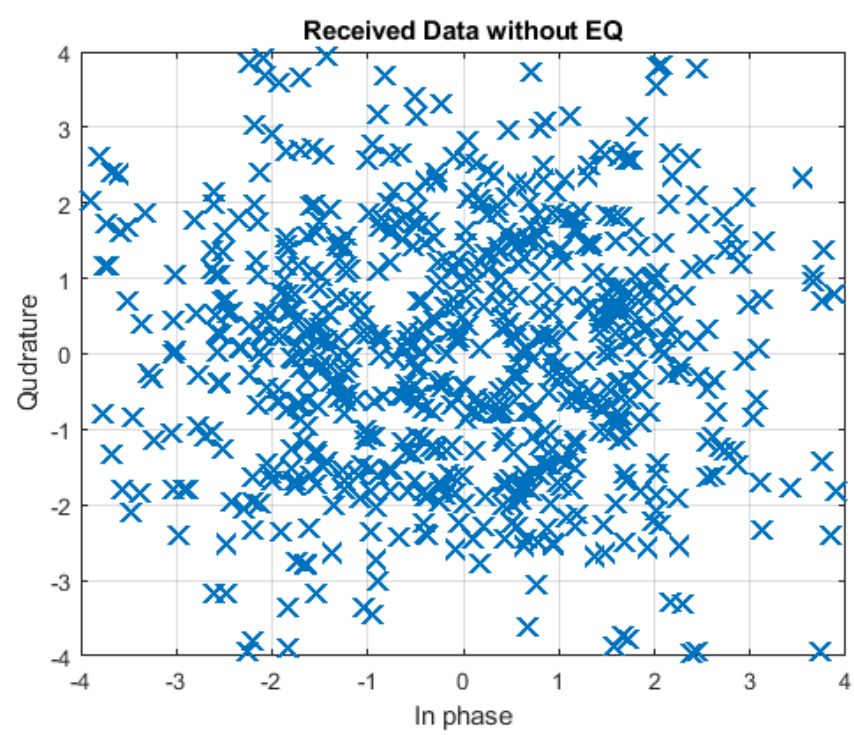

a- received data without using equlizer

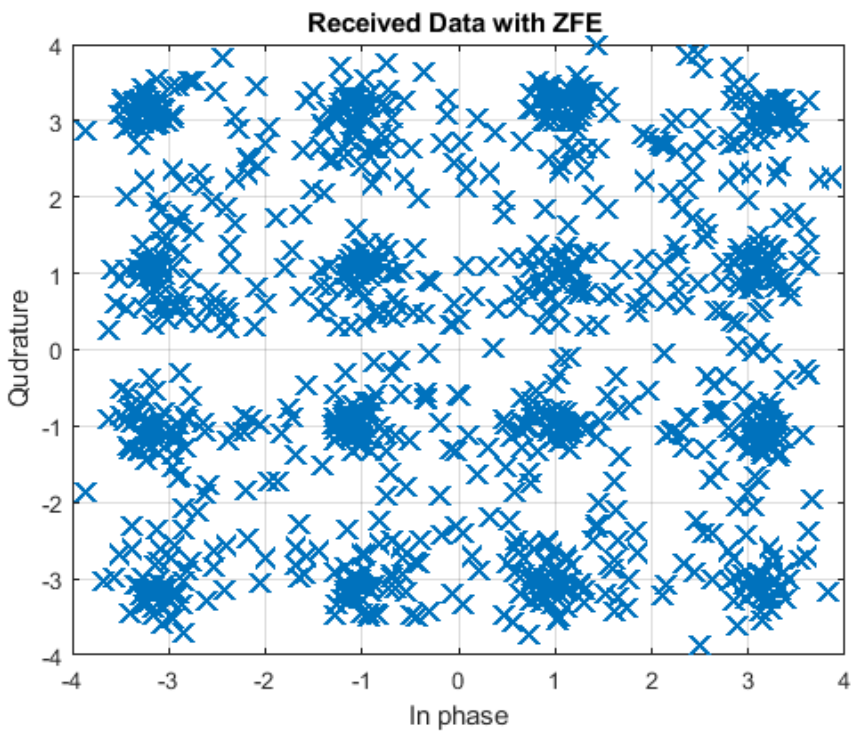

b- received data using $\mathrm{ZF}$ equlizer 


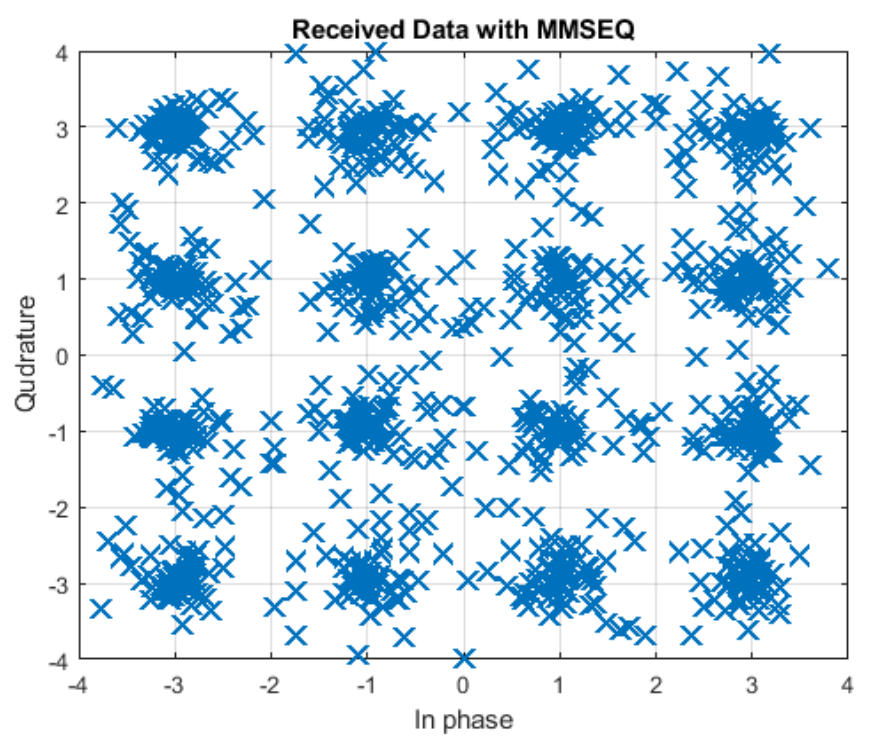

c- received data using MMSE equlizer

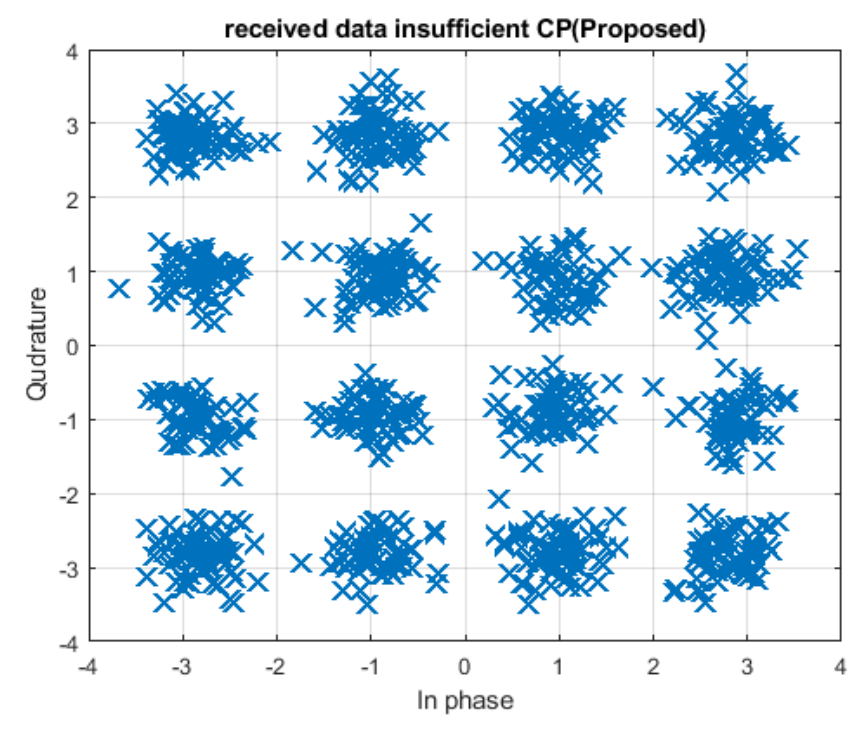

d- received data using LMS equlizer

Fig. 7 Constellation diagram of 16-QAM received data under different types of equalization techniques.

\section{Conclusion:}

An OFDM system with insufficient $\mathrm{CP}$ and LMS equalizer to overcome the multipath channel effects is successfully implemented in this paper. The channel under test has four multipath signals with the presence of Gaussian noise. The block size is 128 and $\mathrm{t}_{\mathrm{s}}$ equal $1 \mu \mathrm{s}$, so the overall time for each block is $128 \mu \mathrm{s}$, the ISI certainly occurs because the multi path signal has twelve samples and the CP has only 6 samples. The results reveal that the proposed method is superior to the MMSE and the $\mathrm{ZF}$ equalization methods by comparing their BER performance and constellation diagrams for 8-QAM and 16-QAM digital modulations. In fig. $4 \mathrm{a}$ and fig. $4 \mathrm{~b}$ because of using semi log graph the small difference could be noticeable. The results also exhibit that the proposed equalization method has OFDM performance close to that when the hypothetical sufficient CP is used (40\% of the data length), which requires noticeably reduced data rate in its transmission.

\section{REFERENCES}

[1] A.Al-Dweik, F.Kalbat, S.Muhaidat, O.Filio, "Robust MIMO-OFDM System for FrequencySelective Mobile Wireless Channels," IEEE Transactions on Vehicular Technology, Vol.64, No.5, pp.1739-1749, MAY 2015.

[2] A.Peled, A.Ruiz, "Frequency domain data transmission using reduced computational complexity algorithms," ICASSP 80. IEEE International Conference on Acoustics, Speech and Signal Processing, pp.964-967, 1980.

[3] Y.S.Cho, J.Kim, W.Yang, C.Kang, MIMOOFDM wireless communications with MATLAB, John Wiley and Sons, Asia Singapore, 2010. 
[4] R.Prasad, OFDM for Wireless Communications Systems, Artech House, Boston London, 2004.

[5] S.Zhou, Z.Wang, OFDM for Underwater Acoustic Communications, First Edition, John Wiley and Sons, United Kingdom, 2014.

[6] H.Kim, Wireless Communications Systems Design, First Edition, John Wiley and Sons, United Kingdom, 2015

[7] K.Choi, H.Liu, Problem-Based Learning in Communication Systems Using MATLAB and Simulink, First Edition, John Wiley and Sons , Hoboken New Jersey, 2016.

[8] B. Kamali, An IEEE 802.16 Standard-Based Technology for the Next Generation of Air Transportation Systems, First Edition, John Wiley and Sons , Hoboken USA, 2019.

[9] A.Jaradat, J.Hamamreh, H.Arslan, "Modulation Options for OFDM-Based Waveforms: Classification, Comparison,and Future," IEEE Access, Vol.7, pp.17263-17278, 2019.

[10] G. Charalabopoulos, P.Stavroulakis, A.H.Aghvami, "A Frequency-Domain Neural Network Equalizer for OFDM," IEEE GLOBECOM IEEE Global Telecommunications Conference, Vol.7, pp.571-575, 2003.

[11] S.Chen, T.Yao, "FEQ for OFDM Systems with Insufficient CP," The 14th IEEE International Symposium on Persona1 Indoor and Mobile Radio Communication Proceedings,Vol.3, pp.550-553, 2003.

[12] W.Zhong, Z.Mao, "Tentative Decision Based Low Complexity Equalization for OFDM Systems with Insufficient Cyclic Prefix," 5th International Conference on Information Communications \& Signal Processing, pp.116119, 2005.

[13] J.Lim, C.Choi, G.Im, "MIMO-OFDM with Insufficient Cyclic Prefix," IEEE COMMUNICATIONS LETTERS, Vol.10, No.5, pp.356-358, MAY 2006.
[14] M.Nisar, H.Nottenstriner, “On Channel Estimation and Equalization of OFDM Systems with Insufficient Cyclic Prefix," IEEE 65th Vehicular Technology Conference - VTC2007Spring, pp.1445-1449, 2007.

[15] K.Shimezawa, T.Yoshimoto, R.Yamada, N.Okamoto, K.Imamura, “A Novel SC/MMSE Turbo Equalization for Multicarrier Systems with Insufficient Cyclic Prefix," IEEE 19th International Symposium on Personal Indoor and Mobile Radio Communications, 2008.

[16] T.Pham, T.Le-Ngoc, G.Woodward, P.Martin, K.Phan, "Equalization for MIMO-OFDM Systems with Insufficient Cyclic Prefix," IEEE 83rd Vehicular Technology Conference (VTC Spring), 2016.

[17] G.Parsaee, A.Yarali, H.Ebrahimzad, "MMSE-DFE Equalizer Design for OFDM Systems with Insufficient Cyclic Prefix," IEEE 60th Vehicular Technology Conference, Vol.6, pp.3828-3832, 2004.

[18] W.Henkel, G.Taubock, P.Odling, P.Borjesson, N.Petersson, "The Cyclic Prefix of OFDM/DMT - An Analysis," International Zurich Seminar on Broadband Communications, pp.22-1 - 22-3, 2002.

[19] J.Zhu, W.Ser, A.Nehorai, "Channel Equalization for DMT with Insufficient Cyclic Prefix," Conference Record of the ThirtyFourth Asilomar Conference on Signals Systems and Computers, Vol.2, pp.951-955, 2000.

[20] A.Poularikas, Fundamentals of Least Mean Squares with MATLAB, Taylor and Francis Group, Boca Raton, 2015.

[21] L.Samara，A.Al-Abbasi， R.Hamila， N.AlDhahir, "Sparse Equalizers for OFDM Signals with Insufficient Cyclic Prefix," IEEE Access, Vol.6, pp.11076-11085, 2017.

[22] M.Failli, Cost 207:Digital land mobile radio communications, European Communities / Union, Brussels Luxembourg, 1989. 